\title{
Results of the implantation of bone-anchored hearing aids in patients with treacher-collins syndrome
}

\author{
Alexandra Kolontai de Sousa Oliveira', Lília Pereira Abreu Ferro', Jaiede Nicacio da Silva², Daniel Mochida Okada³. \\ 1) Resident Doctor in the Department of Otolaryngology at the Institute of Medical Assistance to the State's Public Service, Sao Paulo. \\ 2) Speech Therapist at the Institute of Medical Assistance to the State's Public Service, Sao Paulo. \\ 3) Master of Health Sciences. Physician Assistant Hospital Service, State of São Paulo. \\ Institution: Hospital do Servidor Publico Estadual de São Paulo. \\ São Paulo/SP - Brazil \\ Mailing address: Alexandra Kolontai de Sousa Oliveira - Rua Afonso Celso 718, Apto. 21 - Vila Mariana - São Paulo / SP - Brazil - Zip code: 04119-903 - Telephone: \\ (+55 11) 5088-8479 - E-mail: alekolon@yahoo.com.br \\ Article received on July 31, 2011. Article accepted on February 6, 2012
}

\section{SUMMARY}

Introduction: Treacher-Collins syndrome is characterized by craniofacial malformations, narrowing of the external auditory canal (EAC), and, in 30\% of cases, agenesis of the canal and ossicular chain defects. The use of hearing aids (HA) is not possible in cases in which agenesis or stenosis of the EAC accompanies conductive deafness. In contrast, bone conduction implants such as the Bone Anchored Hearing Aid (BAHA $\left.{ }^{\circledR}\right)$ allow direct stimulation of the cochlea and are thus superior to conventional hearing aids in cases of severe conductive hearing loss.

Objective: To present 2 cases of patients with Treacher-Collins syndrome who underwent implantation of BAHA ${ }^{\circledR}$

Cases Reports: The first patient was a 52-year-old woman diagnosed with Treacher-Collins syndrome who presented with severe bilateral mixed hearing loss and a history of unsuccessful previous use of a bone contact conduction device. The BAHA ${ }^{\circledR}$ implantation was uneventful, and the post-operative results were good. The second patient was a 14-year-old girl who was also diagnosed with Treacher-Collins Syndrome with bilateral moderate conductive hearing loss by audiometry. The use of a bone vibrator contact device did not improve her hearing; however, implantation of a BAHA ${ }^{\circledR}$ resulted in a decreased gap postoperatively. Final comments: $\mathrm{BAHA}^{\circledR}$ hearing devices provide adequate rehabilitation and consequent improvement of the quality of life in patients with Treacher-Collins syndrome.

Keywords: mandibulofacial dysostosis; hearing loss, conductive; bone conduction.

\section{INTRODUCTION}

Treacher-Collins syndrome is characterized by craniofacial deformities arising from the first and second branchial arches and nasal placodes, probably as a result of disruption of neural crest cell development (1).

The pinna is often distorted with excessive wrinkling and may have low implantation. The external auditory canal (EAC) is narrowed, and 30\% of cases present with agenesis of the EAC or defects in the ossicular chain, both of which are accompanied by conductive hearing loss. Profound sensorineural hearing loss (SNHL) is rare in patients with this syndrome (2).

The use of individual hearing aids (HA) is impossible in these cases due to the inability to stimulate the inner ear by airway hearing conduction. Reconstructive surgery of the pinna and the EAC is extremely technically difficult, and the results have not been encouraging (2).

The Bone Anchored Hearing Aid (BAHA ${ }^{\circledR}$ ) system, developed by Tjellstrom in 1977, has several advantages over conventional prosthetic osseous conduction devices and has been used in over 20,000 patients worldwide. The vibration is absorbed by the skull and directly stimulates the cochlea, making the BAHA ${ }^{\circledR}$ system superior to conventional hearing aids in cases of sensorineural and conductive (3).

Osseointegrated implants were first introduced into clinical practice in Sweden in 1977 by Tjellstrom, using Branemark system deployment, to treat patients with conductive or mixed hearing loss that could not be addressed with conventional hearing aids. The procedure is a 2-step process with an average delay of 3 months between the implantation and placement of the processor. The osseointegration of the implant within that period ensures the stability of the implant and is thus critical to the success of the BAHA ${ }^{\circledR}$ system $(9,12)$.

The use of hearing aids or surgical techniques can treat conductive or mixed hearing loss from many causes. However, patients with agenesis or stenosis of the EAC, as well as some with chronic suppurative otitis media (CSOM) or chronic suppurative otitis externa (CSOE) or who have undergone open mastoidectomy, may be unable to adapt to hearing aids; alternatively, the surgical 
results may be poor due to inability of the airway to stimulate the inner ear or poor adaptation, which can generate problems such as discomfort and otorrhea. Tiara-type prosthetic bone conduction devices would be a better option in such cases. However, these implants also have drawbacks that can lead to the patient's abandoning their use. These include skin irritation due to the constant pressure on the local device support, poor aesthetic appeal, and the difficulty of keeping the easily removable tiara in place in children (7).

The $\mathrm{BAHA}^{\circledR}$ system is an interesting alternative in these cases and has advantages over conventional bone conduction devices. It produces excellent results in cases of conductive hearing loss, but its use is limited in cases of associated deafness (12). The implantation surgery is a safe procedure with few major complications.

\section{LITERATURE REVIEW}

The use of osseointegrated implants was first introduced in 1977 in Sweden by Tjellstrom, using Branemark system deployment, to treat patients with conductive or mixed hearing loss that could not be addressed with conventional hearing aids. The procedure is a 2-step process, with an average delay of 3 months between the implantation and placement of the processor. The osseointegration of the implant during this time ensures the implant's stability and is thus critical to the success of the BAHA $^{\circledR}$ System $(9,11)$.

The use of hearing aids or surgical techniques can treat conductive or mixed hearing loss due to many causes. However, patients with agenesis or stenosis of the EAC, as well as some with CSOM or CSOE externa or who have undergone open mastoidectomy, cannot adapt to hearing aids; alternatively, the surgical results may be poor due to the lack of stimulation of the inner ear by air or bad adaptation, which can lead to problems such as discomfort and otorrhea. The tiara type of bone conduction hearing device would be a better option in these cases. However, these implants also have drawbacks that can lead to patients' abandoning their use. These include skin irritation due to the constant pressure on the local support of the device, poor aesthetic appeal, and the difficulty of keeping the easily removed tiara in place in children (3).

The $\mathrm{BAHA}^{\oplus}$ system is an interesting alternative in these cases and also has other advantages over conventional bone conduction (BC) devices. It produces excellent results in cases of conductive hearing loss; however, its usefulness is limited in cases of associated deafness (12). The surgical implantation is a safe procedure with few major complications.

\section{Case Report}

CASE 1: S.A.T. was a 52-year-old woman who complained of bilateral hearing loss since birth and had been diagnosed with Treacher-Collins syndrome. Physical examination showed atresia of the EAC. Audiometry showed mixed hearing loss, and computed tomography showed severe bilateral temporal bone atresia at the EAC without changes in the middle or inner ear. (Figure 1) The patient had used a tiara-type bone contact device for 10 years but had recently noted worsening in her hearing. After jointly assessing the case with speech therapists and obtaining the patient's consent, the clinicians opted for a BAHA ${ }^{\circledR}$ implant in the left mastoid. The surgical procedure was performed via a vertical incision technique and was uneventful. (Figure 2) One late complication occurred in that skin grew over the titanium implant; this was solved by simple resection in the doctor's office. A 4-month period was allowed for osseointegration. The patient states that she is satisfied with the results and has noted significant improvement in her work and in everyday activities such as watching television, talking with friends, answering calls, and listening to music. She uses the prosthesis 12 hours per day with the drawback that the battery life is short (13 days). The audiometric results obtained with the use of the prosthesis showed a postoperative reduction in the speech reception threshold (SRT) by $35 \mathrm{~dB}$ and a speech recognition rate of $65 \%$ at $92 \mathrm{~dB}$ (Figure 4).

CASE 2: KVF was a 14-year-old girl who was diagnosed with Treacher-Collins and who complained of poor performance at school. Physical examination showed complete agenesis of the EAC. Audiometry revealed

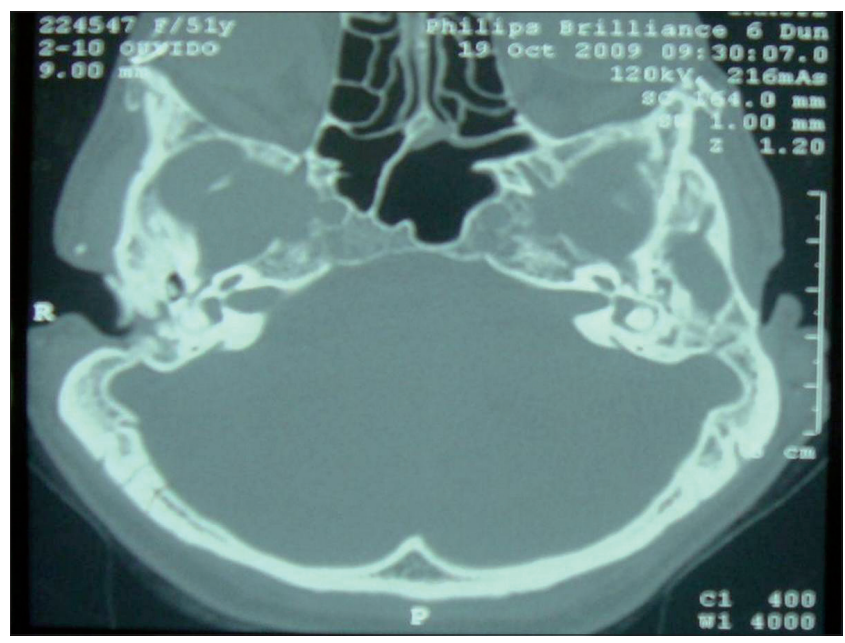

Figure 1. Computed tomography of the temporal bone, axial section, showing atresia of the external auditory canal. 


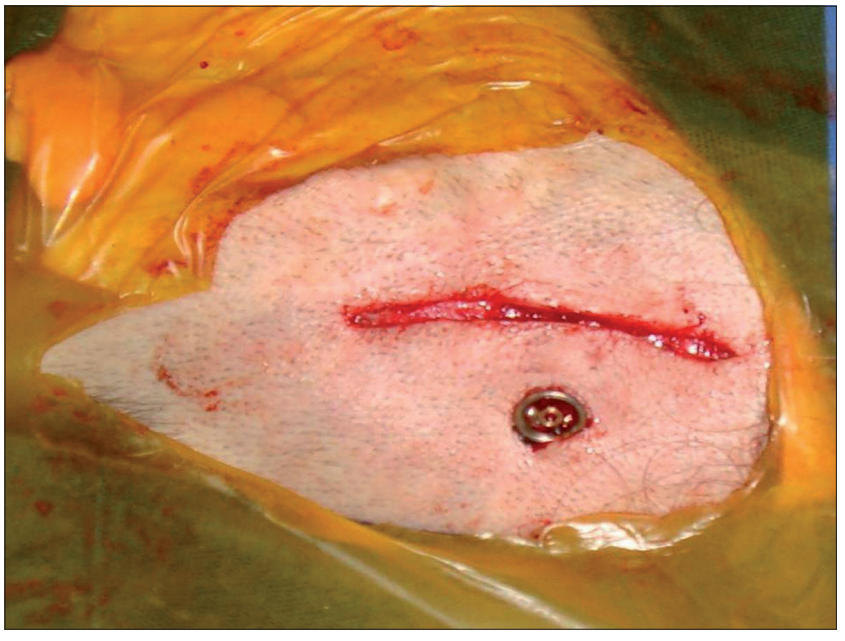

Figure 2. Intra-operative photo showing the titanium screw implanted in the cortical mastoid.

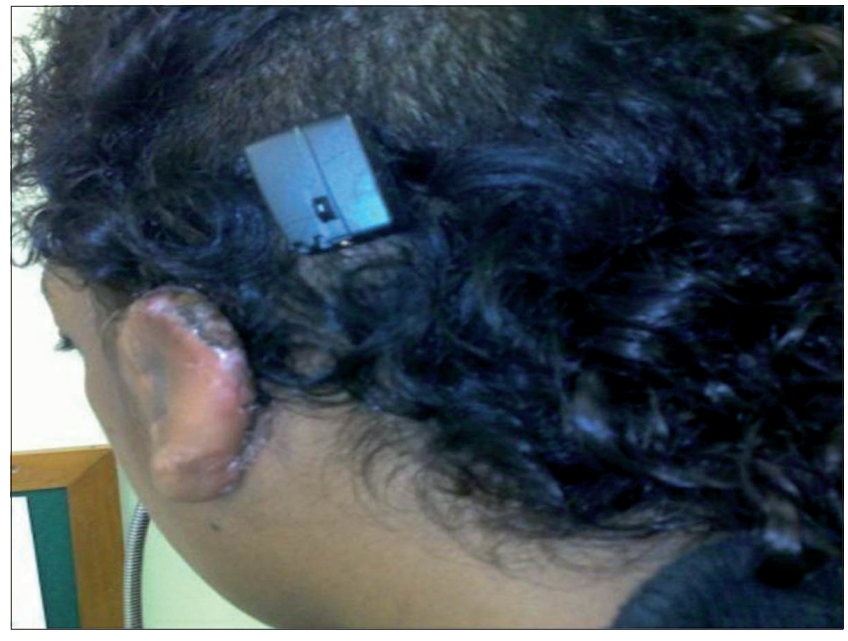

Figure 3. Speech processor coupled.

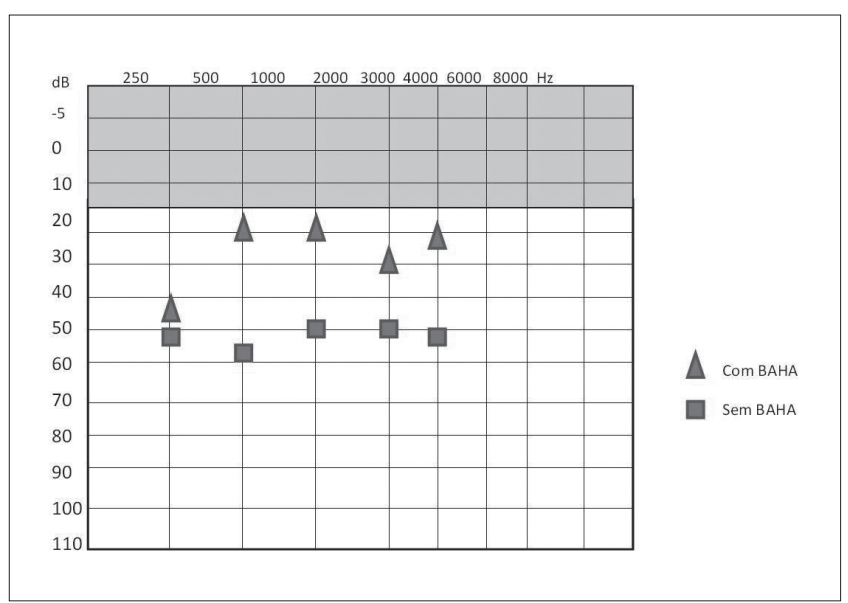

Figure 5. Post-procedure free field audiometry results with and without $\mathrm{BAHA}^{\circledR}$ in case 2 .

learned to maintain proper hygiene and care of the implant.

\section{Discussion}

The bone conduction prosthesis was developed by Tjellstrom in 1977. The implant unit consists of a titanium screw that is implanted in the mastoid cortical bone via an external approach. This unit is separate from the processor that captures the sound energy of the environment and transforms it into mechanical energy, which is translated into vibration by the stimulation of the cranial cortical bone. 
The tiara-type bone vibration prosthesis was previously the only recourse in cases of conductive hearing loss that did not benefit from the use of a hearing aid (HA), as in cases of congenital deformities of the ear and CAE. However, this device has some disadvantages that prevent its suitability in many cases, such as discomfort from the constant pressure exerted by the tiara, poor sound quality due to attenuation of high frequency sounds by the skin, positioning difficulties in children, and aesthetic considerations. The bone conduction prosthesis avoids some of these problems: it does not put any pressure on the skin, produces better sound quality, remains firmly positioned in the temporal bone, and is more aesthetically acceptable.

The limitations of prosthetic hearing devices in cases of sensorineural dysacousia require careful assessment and selection of the candidate patient. There are no limitations to their use in patients with conductive hearing loss as the auditory stimulation bypasses the airway (2). In order to avoid complications, tomography of the temporal bones should always be performed prior to surgery in order to assess the thickness of the skull and the positions of the facial nerve and sigmoid sinus (5).

The placement of bone conduction prostheses is a low-risk surgery that provides an excellent alternative for patients with congenital deformities, such as those with Treacher-Collins syndrome. Surgical treatment of atresia at the EAC produces poor results in many cases. The potential major complications of bone conduction implant placement include restenosis, lateralization of the facial nerve, facial nerve damage, and temporomandibular joint dysfunction (7). The incidence of restenosis is approximately 20 to $50 \%$ (8). When the implant is to be unilateral, it should be placed on the side with the best sensorineural threshold. Studies have shown that bilateral BAHA improves patient satisfaction in cases with symmetrical thresholds $(3,7)$.

The process of osseointegration produces a direct structural and functional connection between the bone and implant surface without intermediary fibrous tissue. The osseointegration reflects the healing of the endosteum, a process involving hematoma formation buffer, buffer resolution, migration of osteogenic cells, and bone formation. The process of bone healing adjacent to the implant is identical to physiological healing. Postoperatively, ankylosis occurs at the interface at which the implant contacts the bone surface and is maintained through a dynamic equilibrium after osseointegration (10).

The major potential intra-operative problems are exposure of the dura mater, exposure of the sigmoid sinus, and opening of the mastoid cells. In children, the thinness of the skull may result in incomplete deployment of the titanium pin. The potential post-operative complications include flap necrosis or redness, irritation, or granulation of the flap, osseointegration failure, trauma, and surgical site infection. The frequency of implant loss is approximately $5 \%$ (8) and is slightly higher in children, in whom it ranges from 7.5 to $15 \%(2,4)$. Major complications such as cerebral abscesses are rare (6).

BAHA $^{\circledR}$ implantation is a low-risksurgery that provides an excellent alternative for patients with congenital deformities, such as those occurring in cases of TreacherCollins syndrome. Surgical treatment of atresia of the EAC does not produce good results in many cases.

\section{Final Considerations}

BAHA $^{\star}$ systems provide good rehabilitation and consequently improve the quality of life of patients with Treacher-Collins syndrome.

\section{REFERENCES}

1. Posnick JC, Ruiz RL. Treacher Collins Syndrome: Current Evaluation, Treatment, and Future Directions. Cleft Palate Craniofac J. 2000;37(5):434-5.

2. Zhang ZMD, Niu FMD, Tang XMD, Yu, Bing MD, Liu JMD, Gui L MD. Staged Reconstruction for Adult Complete Treacher Collins Syndrome. J Craniofac Surg. 2009;20(5):1433-8.

3. Wazen JJ, Young DL, Farrugia MC, Chandrasekhar SS, Ghossaini SN, Borik J, Soneru C, Spitzer JB. Successes and Complications of the Baha System. Otol Neurotol. 2008;29(8):1115-9.

4. Shirazi MA, Marzo SJ, Leonetti JP. Perioperative Complications With the Bone Anchored Hearing Aid. Otolaryngol Head Neck Surg. 2006;134(2):236-9.

5. Mylanus EAM, Van Der Pouw CTM, Snik AFM, Cremers CWRJ. An Intraindividual comparison of the BAHA and air conduction hearing aids. Arch Otolaryngol Head NeckSurg. 1998;(124):271-6.

6. Dutt SN, McDermott AL, Jelbert A, Reid AP, Proops DW. The Glasgow benefit inventory in the evaluation of patient satisfaction with the bone anchored hearing aid: quality of life issues. J Laryngol Otol Suppl. 2002 Jun;(28):7-14.

7. Wazen JJ, Gupta R, Ghossaini S, Spitzer J, Farrugia M, 
Tjellstrom A. Osseointegration Timing for Baha System Loading. Laryngoscope. 2007 May;117(5):794-6.

8. Hakansson B, Tjellstrom A, Rosenhall U. Hearing thresholds with direct bone conduction versus conventional bone conduction. Scand Audiol. 1984;13(1):3-13.

9. Tjellstrom A, Lindstrom J, Hallen O, et al. Osseointegrated titanium implants in the temporal bone. Am J Otol 1981;(2):304-10.

10. Wazen JL, Grupta R, Ghossaini S, Spitzer J, Farrugia M,
Tjellstrom A. Osseointegration Timing for Baha System Loading. Laryngoscope 2007;117(5):794-6.

11. WazenJJ, Caruso M, Tjellstrom A. Long-term results with the titanium bone anchored hearing aid: the U.S. experience. Am J Otol. 1998 Nov;19(6):737-41.

12. Van Der Pouw CTM, Mylanus EAM, Cremers CWRJ. Percutaneus implant in the temporal bone for securing a bone conductor: surgical methods and results. Ann Otol Rhinol Laryngol. 1999;108(6):532-7. 